\title{
Refinements of Aczél-Type Inequality and Their Applications
}

\author{
Jingfeng Tian' ${ }^{1}$ and Wen-Li Wang ${ }^{2}$ \\ ${ }^{1}$ College of Science and Technology, North China Electric Power University, Baoding, Hebei 071051, China \\ ${ }^{2}$ Department of Information Engineering, China University of Geosciences Great Wall College, Baoding 071000, China
}

Correspondence should be addressed to Jingfeng Tian; tianjfhxm_ncepu@163.com

Received 22 April 2014; Revised 8 June 2014; Accepted 9 June 2014; Published 26 June 2014

Academic Editor: Shanhe Wu

Copyright (c) 2014 J. Tian and W.-L. Wang. This is an open access article distributed under the Creative Commons Attribution License, which permits unrestricted use, distribution, and reproduction in any medium, provided the original work is properly cited.

We present some new sharpened versions of Aczél-type inequality. Moreover, as applications, some refinements of integral type of Aczél-type inequality are given.

\section{Introduction}

Let $n$ be a positive integer, and let $a_{i}, b_{i}(i=1,2, \ldots, n)$ be real numbers such that $a_{1}^{2}-\sum_{i=2}^{n} a_{i}^{2}>0$ or $b_{1}^{2}-\sum_{i=2}^{n} b_{i}^{2}>0$. Then, the famous Aczél inequality [1] can be stated as follows:

$$
\left(a_{1}^{2}-\sum_{i=2}^{n} a_{i}^{2}\right)\left(b_{1}^{2}-\sum_{i=2}^{n} b_{i}^{2}\right) \leq\left(a_{1} b_{1}-\sum_{i=2}^{n} a_{i} b_{i}\right)^{2} .
$$

Aczél's inequality plays a very important role in the theory of functional equations in non-Euclidean geometry. Due to the importance of Aczél's inequality (1), it has received considerable attention by many authors and has motivated a large number of research papers giving it various generalizations, improvements, and applications (see [2-21] and the references therein).

In 1959, Popoviciu [10] first obtained an exponential extension of the Aczél inequality as follows.

Theorem B. Let $p \geq q>1,(1 / p)+(1 / q)=1$, and let $a_{i}$, $b_{i}(i=1,2, \ldots, n)$ be positive numbers such that $a_{1}^{p}-\sum_{i=2}^{n} a_{i}^{p}>$ 0 and $b_{1}^{q}-\sum_{i=2}^{n} b_{i}^{q}>0$. Then

$$
\left(a_{1}^{p}-\sum_{i=2}^{n} a_{i}^{p}\right)^{1 / p}\left(b_{1}^{q}-\sum_{i=2}^{n} b_{i}^{q}\right)^{1 / q} \leq a_{1} b_{1}-\sum_{i=2}^{n} a_{i} b_{i} .
$$

Later, in 1982, Vasić and Pečarić [16] established the following reversed version of inequality (2).
Theorem C. Let $q<0, p>0,(1 / p)+(1 / q)=1$, and let $a_{i}$, $b_{i}(i=1,2, \ldots, n)$ be positive numbers such that $a_{1}^{p}-\sum_{i=2}^{n} a_{i}^{p}>$ 0 and $b_{1}^{q}-\sum_{i=2}^{n} b_{i}^{q}>0$. Then

$$
\left(a_{1}^{p}-\sum_{i=2}^{n} a_{i}^{p}\right)^{1 / p}\left(b_{1}^{q}-\sum_{i=2}^{n} b_{i}^{q}\right)^{1 / q} \geq a_{1} b_{1}-\sum_{i=2}^{n} a_{i} b_{i} .
$$

In another paper, Vasić and Pečarić [15] generalized inequality (2) in the following form.

Theorem D. Let $a_{r j}>0, \beta_{j}>0, a_{1 j}^{\beta_{j}}-\sum_{r=2}^{n} a_{r j}^{\beta_{j}}>0, r=$ $1,2, \ldots, n, j=1,2, \ldots, m$, and let $\sum_{j=1}^{m}\left(1 / \beta_{j}\right) \geq 1$. Then

$$
\prod_{j=1}^{m}\left(a_{1 j}^{\beta_{j}}-\sum_{r=2}^{n} a_{r j}^{\beta_{j}}\right)^{1 / \beta_{j}} \leq \prod_{j=1}^{m} a_{1 j}-\sum_{r=2}^{n} \prod_{j=1}^{m} a_{r j} .
$$

In 2012, Tian [13] presented the reversed version of inequality (4) as follows.

Theorem E. Let $a_{r j}>0, \beta_{1} \neq 0, \beta_{j}<0(j=2,3, \ldots, m)$, $\sum_{j=1}^{m}\left(1 / \beta_{j}\right) \leq 1, a_{1 j}^{\beta_{j}}-\sum_{r=2}^{n} a_{r j}^{\beta_{j}}>0, r=1,2, \ldots, n, j=$ $1,2, \ldots, m$. Then

$$
\prod_{j=1}^{m}\left(a_{1 j}^{\beta_{j}}-\sum_{r=2}^{n} a_{r j}^{\beta_{j}}\right)^{1 / \beta_{j}} \geq \prod_{j=1}^{m} a_{1 j}-\sum_{r=2}^{n} \prod_{j=1}^{m} a_{r j} .
$$

Moreover, in [13] Tian established an integral type of inequality (5). 
Theorem F. Let $\beta_{1}>0, \beta_{j}<0(j=2,3, \ldots, m)$, $\sum_{j=1}^{m}\left(1 / \beta_{j}\right)=1$, let $t_{j}>0(j=1,2, \ldots, m)$, and let $f_{j}(x)(j=$ $1,2, \ldots, m)$ be positive Riemann integrable functions on $[a, b]$ such that $t_{j}^{\beta_{j}}-\int_{a}^{b} f_{j}^{\beta_{j}}(x) d x>0$. Then

$$
\prod_{j=1}^{m}\left(t_{j}^{\beta_{j}}-\int_{a}^{b} f_{j}^{\beta_{j}}(x) d x\right)^{1 / \beta_{j}} \geq \prod_{j=1}^{m} t_{j}-\int_{a}^{b} \prod_{j=1}^{m} f_{j}(x) d x .
$$

Remark 1. In fact, the integral form of inequality (4) is also valid; that is, one has the following.

Theorem G. Let $\beta_{j}>0(j=1,2, \ldots, m), \sum_{j=1}^{m}\left(1 / \beta_{j}\right)=1$, let $t_{j}>0(j=1,2, \ldots, m)$, and let $f_{j}(x)(j=1,2, \ldots, m)$ be positive Riemann integrable functions on $[a, b]$ such that $t_{j}^{\beta_{j}}-$ $\int_{a}^{b} f_{j}^{\beta_{j}}(x) d x>0$. Then

$$
\prod_{j=1}^{m}\left(t_{j}^{\beta_{j}}-\int_{a}^{b} f_{j}^{\beta_{j}}(x) d x\right)^{1 / \beta_{j}} \leq \prod_{j=1}^{m} t_{j}-\int_{a}^{b} \prod_{j=1}^{m} f_{j}(x) d x .
$$

The main purpose of this work is to give new refinements of inequalities (4) and (5). As applications, new refinements of inequalities (6) and (7) are also given.

\section{Refinements of Aczél-Type Inequality}

In order to present our main results, we need some lemmas as follows.

Lemma 2 (see [6]). Let $a_{i}, x_{i}(i=1,2, \ldots, n)$ be real numbers such that $a_{i} \geq 0$ and $x_{i}>-1$. If $\sum_{i=1}^{n} a_{i} \leq 1$, then

$$
\prod_{i=1}^{n}\left(1+x_{i}\right)^{a_{i}} \leq 1+\sum_{i=1}^{n} a_{i} x_{i}
$$

If either $a_{i} \geq 1(i=1,2, \ldots, n)$ or $a_{i} \leq 0(i=1,2, \ldots, n)$ and if all $x_{i}$ are positive or negative with $x_{i}>-1$, then the reverse inequality of (8) holds.

Lemma 3 (see [15]). Let $a_{i j}>0(i=1,2, \ldots, n, j=$ $1,2, \ldots, m)$.

(a) If $\lambda_{j} \geq 0$ and if $\sum_{j=1}^{m} \lambda_{j} \geq 1$, then

$$
\sum_{i=1}^{n} \prod_{j=1}^{m} a_{i j}^{\lambda_{j}} \leq \prod_{j=1}^{m}\left(\sum_{i=1}^{n} a_{i j}\right)^{\lambda_{j}}
$$

(b) If $\lambda_{j} \leq 0(j=1,2, \ldots, m)$, then

$$
\sum_{i=1}^{n} \prod_{j=1}^{m} a_{i j}^{\lambda_{j}} \geq \prod_{j=1}^{m}\left(\sum_{i=1}^{n} a_{i j}\right)^{\lambda_{j}}
$$

(c) If $\lambda_{1}>0, \lambda_{j} \leq 0(j=2,3, \ldots, m)$, and $\sum_{j=1}^{m} \lambda_{j} \leq 1$, then

$$
\sum_{i=1}^{n} \prod_{j=1}^{m} a_{i j}^{\lambda_{j}} \geq \prod_{j=1}^{m}\left(\sum_{i=1}^{n} a_{i j}\right)^{\lambda_{j}}
$$

Lemma 4 (see $[18]$ ). Let $0 \leq x<1, \alpha>0$. Then

$$
(1-x)^{1 / \alpha} \leq 1-\frac{x}{\max \{\alpha, 1\}}
$$

Lemma 5. Let $0<\beta_{1} \leq \beta_{2} \leq \cdots \leq \beta_{m}, \sum_{j=1}^{m}\left(1 / \beta_{j}\right) \geq 1$, $m \geq 2$, let $0<x_{j}<1(j=1,2, \ldots, m)$, and let $\xi(m)=$ $\left\{\begin{array}{ll}m / 2 & \text { if } m \text { even } \\ (m-1) / 2 & \text { if } m \text { odd }\end{array}\right.$.

Then

$$
\begin{aligned}
& \prod_{j=1}^{m}\left(1-x_{j}^{\beta_{j}}\right)^{1 / \beta_{j}}+\prod_{j=1}^{m} x_{j} \\
& \quad \leq 1-\frac{1}{\xi(m)} \times \sum_{j=1}^{\xi(m)}\left[\frac{1}{\max \left\{\beta_{2 j}, 1\right\}}\left(x_{2 j}^{\beta_{2 j}}-x_{2 j-1}^{\beta_{2 j-1}}\right)^{2}\right] .
\end{aligned}
$$

Proof. From the assumptions we have that

$$
\begin{gathered}
\frac{1}{\beta_{1}} \geq \frac{1}{\beta_{2}} \geq \cdots \geq \frac{1}{\beta_{m-1}} \geq \frac{1}{\beta_{m}}>0, \\
\frac{1}{\beta_{j}}-\frac{1}{\beta_{j+1}} \geq 0 \quad(j=1,2, \ldots, m-1) .
\end{gathered}
$$

Case (I) (let $m$ be even). In view of $\left(1 / \beta_{1}-1 / \beta_{2}\right)+1 / \beta_{2}+1 / \beta_{2}+$ $\left(1 / \beta_{3}-1 / \beta_{4}\right)+1 / \beta_{4}+1 / \beta_{4}+\cdots+\left(1 / \beta_{m-1}-1 / \beta_{m}\right)+1 / \beta_{m}+$ $1 / \beta_{m}=1 / \beta_{1}+1 / \beta_{2}+\cdots+1 / \beta_{m} \geq 1$ by using inequality (9), we get

$$
\begin{aligned}
& \prod_{j=1}^{m / 2}\left[1-\left(x_{2 j}^{\beta_{2 j}}-x_{2 j-1}^{\beta_{2 j-1}}\right)^{2}\right]^{1 / \beta_{2 j}} \\
& =\prod_{j=1}^{m / 2}\left\{\left[\left(1-x_{2 j-1}^{\beta_{2 j-1}}\right)+x_{2 j}^{\beta_{2 j}}\right]^{1 / \beta_{2 j}}\right. \\
& \times\left[\left(1-x_{2 j}^{\beta_{2 j}}\right)+x_{2 j-1}^{\beta_{2 j-1}}\right]^{1 / \beta_{2 j}} \\
& \left.\times\left[\left(1-x_{2 j-1}^{\beta_{2 j-1}}\right)+x_{2 j-1}^{\beta_{2 j}-1}\right]^{1 / \beta_{2 j-1}-1 / \beta_{2 j}}\right\} \\
& =\left[\left(1-x_{1}^{\beta_{1}}\right)+x_{2}^{\beta_{2}}\right]^{1 / \beta_{2}}\left[\left(1-x_{2}^{\beta_{2}}\right)+x_{1}^{\beta_{1}}\right]^{1 / \beta_{2}} \\
& \times\left[\left(1-x_{1}^{\beta_{1}}\right)+x_{1}^{\beta_{1}}\right]^{1 / \beta_{1}-1 / \beta_{2}} \\
& \times\left[\left(1-x_{3}^{\beta_{3}}\right)+x_{4}^{\beta_{4}}\right]^{1 / \beta_{4}}\left[\left(1-x_{4}^{\beta_{4}}\right)+x_{3}^{\beta_{3}}\right]^{1 / \beta_{4}} \\
& \times\left[\left(1-x_{3}^{\beta_{3}}\right)+x_{3}^{\beta_{3}}\right]^{1 / \beta_{3}-1 / \beta_{4}} \\
& \times\left[\left(1-x_{m-1}^{\beta_{m-1}}\right)+x_{m}^{\beta_{m}}\right]^{1 / \beta_{m}}\left[\left(1-x_{m}^{\beta_{m}}\right)+x_{m-1}^{\beta_{m-1}}\right]^{1 / \beta_{m}}
\end{aligned}
$$




$$
\begin{gathered}
\times\left[\left(1-x_{m-1}^{\beta_{m-1}}\right)+x_{m-1}^{\beta_{m-1}}\right]^{1 / \beta_{m-1}-1 / \beta_{m}} \\
\geq \prod_{j=1}^{m / 2}\left[\left(1-x_{2 j-1}^{\beta_{2 j-1}}\right)^{1 / \beta_{2 j}}\left(1-x_{2 j}^{\beta_{2 j}}\right)^{1 / \beta_{2 j}}\right. \\
\left.\times\left(1-x_{2 j-1}^{\beta_{2 j-1}}\right)^{1 / \beta_{2 j-1}-1 / \beta_{2 j}}\right] \\
+\prod_{j=1}^{m / 2}\left[\left(x_{2 j}^{\beta_{2 j}}\right)^{1 / \beta_{2 j}}\left(x_{2 j-1}^{\beta_{2 j-1}}\right)^{1 / \beta_{2 j}}\right. \\
\left.\quad \times\left(x_{2 j-1}^{\beta_{2 j-1}}\right)^{1 / \beta_{2 j-1}-1 / \beta_{2 j}}\right] \\
=\prod_{j=1}^{m / 2}\left(1-x_{j}^{\beta_{j}}\right)^{1 / \beta_{j}}+\prod_{j=1}^{m} x_{j} .
\end{gathered}
$$

On the other hand, applying Lemma 4 and the arithmetic-geometric means inequality we obtain

$$
\begin{aligned}
& \prod_{j=1}^{m / 2}\left[1-\left(x_{2 j}^{\beta_{2 j}}-x_{2 j-1}^{\beta_{2 j-1}}\right)^{2}\right]^{1 / \beta_{2 j}} \\
& \quad \leq \prod_{j=1}^{m / 2}\left[1-\frac{1}{\max \left\{\beta_{2 j}, 1\right\}}\left(x_{2 j}^{\beta_{2 j}}-x_{2 j-1}^{\beta_{2 j-1}}\right)^{2}\right] \\
& \quad \leq\left\{\frac{2}{m} \sum_{j=1}^{m / 2}\left[1-\frac{1}{\max \left\{\beta_{2 j}, 1\right\}}\left(x_{2 j}^{\beta_{2 j}}-x_{2 j-1}^{\beta_{2 j-1}}\right)^{2}\right]\right\}^{m / 2} \\
& \quad=\left\{1-\frac{2}{m} \sum_{j=1}^{m / 2}\left[\frac{1}{\max \left\{\beta_{2 j}, 1\right\}}\left(x_{2 j}^{\beta_{2 j}}-x_{2 j-1}^{\beta_{2 j-1}}\right)^{2}\right]\right\}^{m / 2} .
\end{aligned}
$$

Applying Lemma 4 again, we get

$$
\begin{aligned}
& \left\{1-\frac{2}{m} \sum_{j=1}^{m / 2}\left[\frac{1}{\max \left\{\beta_{2 j}, 1\right\}}\left(x_{2 j}^{\beta_{2 j}}-x_{2 j-1}^{\beta_{2 j-1}}\right)^{2}\right]\right\}^{m / 2} \\
& \leq 1-\frac{2}{m} \sum_{j=1}^{m / 2}\left[\frac{1}{\max \left\{\beta_{2 j}, 1\right\}}\left(x_{2 j}^{\beta_{2 j}}-x_{2 j-1}^{\beta_{2 j-1}}\right)^{2}\right] .
\end{aligned}
$$

Combining (15), (16), and (17) yields immediately inequality (13).

Case (II) (let $m$ be odd). In view of $\left(1 / \beta_{1}-1 / \beta_{2}\right)+1 / \beta_{2}+$ $1 / \beta_{2}+\left(1 / \beta_{3}-1 / \beta_{4}\right)+1 / \beta_{4}+1 / \beta_{4}+\cdots+\left(1 / \beta_{m-2}-1 / \beta_{m-1}\right)+$
$1 / \beta_{m-1}+1 / \beta_{m-1}+1 / \beta_{m}=1 / \beta_{1}+1 / \beta_{2}+\cdots+1 / \beta_{m} \geq 1$, by using inequality (9), we have

$$
\begin{aligned}
& \prod_{j=1}^{(m-1) / 2}\left[1-\left(x_{2 j}^{\beta_{2 j}}-x_{2 j-1}^{\beta_{2 j-1}}\right)^{2}\right]^{1 / \beta_{2 j}} \\
& =\left\{\prod_{j=1}^{(m-1) / 2}\left[1-\left(x_{2 j}^{\beta_{2 j}}-x_{2 j-1}^{\beta_{2 j-1}}\right)^{2}\right]^{1 / \beta_{2 j}}\right\} \\
& \times\left[\left(1-x_{m}^{\beta_{m}}\right)+x_{m}^{\beta_{m}}\right]^{1 / \beta_{m}} \\
& =\left\{\prod _ { j = 1 } ^ { ( m - 1 ) / 2 } \left\{\left[\left(1-x_{2 j-1}^{\beta_{2 j-1}}\right)+x_{2 j}^{\beta_{2 j}}\right]^{1 / \beta_{2 j}}\right.\right. \\
& \times\left[\left(1-x_{2 j}^{\beta_{2 j}}\right)+x_{2 j-1}^{\beta_{2 j-1}}\right]^{1 / \beta_{2 j}} \\
& \left.\left.\times\left[\left(1-x_{2 j-1}^{\beta_{2 j-1}}\right)+x_{2 j-1}^{\beta_{2 j-1}}\right]^{1 / \beta_{2 j-1}-1 / \beta_{2 j}}\right\}\right\} \\
& \times\left[\left(1-x_{m}^{\beta_{m}}\right)+x_{m}^{\beta_{m}}\right]^{1 / \beta_{m}} \\
& \geq\left\{\prod _ { j = 1 } ^ { ( m - 1 ) / 2 } \left[\left(1-x_{2 j-1}^{\beta_{2 j-1}}\right)^{1 / \beta_{2 j}}\left(1-x_{2 j}^{\beta_{2 j}}\right)^{1 / \beta_{2 j}}\right.\right. \\
& \left.\left.\left(1-x_{2 j-1}^{\beta_{2 j-1}}\right)^{1 / \beta_{2 j-1}-1 / \beta_{2 j}}\right]\right\}\left(1-x_{m}^{\beta_{m}}\right)^{1 / \beta_{m}} \\
& +\left\{\prod _ { j = 1 } ^ { ( m - 1 ) / 2 } \left[\left(x_{2 j}^{\beta_{2 j}}\right)^{1 / \beta_{2 j}}\left(x_{2 j-1}^{\beta_{2 j-1}}\right)^{1 / \beta_{2 j}}\right.\right. \\
& \left.\left.\times\left(x_{2 j-1}^{\beta_{2 j-1}}\right)^{1 / \beta_{2 j-1}-1 / \beta_{2 j}}\right]\right\}\left(x_{m}^{\beta_{m}}\right)^{1 / \beta_{m}} \\
& =\prod_{j=1}^{m}\left(1-x_{j}^{\beta_{j}}\right)^{1 / \beta_{j}}+\prod_{j=1}^{m} x_{j} .
\end{aligned}
$$

On the other hand, applying Lemma 4 and the arithmeticgeometric means inequality we obtain

$$
\begin{aligned}
& \prod_{j=1}^{(m-1) / 2}\left[1-\left(x_{2 j}^{\beta_{2 j}}-x_{2 j-1}^{\beta_{2 j-1}}\right)^{2}\right]^{1 / \beta_{2 j}} \\
& \quad \leq \prod_{j=1}^{(m-1) / 2}\left[1-\frac{1}{\max \left\{\beta_{2 j}, 1\right\}}\left(x_{2 j}^{\beta_{2 j}}-x_{2 j-1}^{\beta_{2 j-1}}\right)^{2}\right]
\end{aligned}
$$




$$
\begin{aligned}
& \leq\left\{\frac { 2 } { m - 1 } \sum _ { j = 1 } ^ { ( m - 1 ) / 2 } \left[1-\frac{1}{\max \left\{\beta_{2 j}, 1\right\}}\right.\right. \\
& \left.\left.\times\left(x_{2 j}^{\beta_{2 j}}-x_{2 j-1}^{\beta_{2 j-1}}\right)^{2}\right]\right\}^{(m-1) / 2} \\
& =\left\{1-\frac{2}{m-1} \sum_{j=1}^{(m-1) / 2}\left[\frac{1}{\max \left\{\beta_{2 j}, 1\right\}}\right.\right. \\
& \left.\left.\times\left(x_{2 j}^{\beta_{2 j}}-x_{2 j-1}^{\beta_{2 j-1}}\right)^{2}\right]\right\}^{(m-1) / 2} .
\end{aligned}
$$

Applying Lemma 4 again, we have

$$
\begin{aligned}
& \left\{1-\frac{2}{m-1} \sum_{j=1}^{(m-1) / 2}\left[\frac{1}{\max \left\{\beta_{2 j}, 1\right\}}\left(x_{2 j}^{\beta_{2 j}}-x_{2 j-1}^{\beta_{2 j-1}}\right)^{2}\right]\right\}^{(m-1) / 2} \\
& \leq 1-\frac{2}{m-1} \sum_{j=1}^{(m-1) / 2}\left[\frac{1}{\max \left\{\beta_{2 j}, 1\right\}}\left(x_{2 j}^{\beta_{2 j}}-x_{2 j-1}^{\beta_{2 j-1}}\right)^{2}\right] .
\end{aligned}
$$

Hence, combining (18), (19), and (20) yields immediately inequality (13).

Similar to the proof of Lemma 5 but using Lemma 2 in place of Lemma 4 , we immediately obtain the following result.

Lemma 6. Let $\beta_{1}>0,0>\beta_{2} \geq \beta_{3} \geq \cdots \geq \beta_{m}, \sum_{j=1}^{m}\left(1 / \beta_{j}\right) \leq$ $1, m \geq 2$, let $0<x_{1}<1, x_{j}>1(j=2,3, \ldots, m)$, and let $\xi(m)=\left\{\begin{array}{ll}m / 2 & \text { if } m \text { even } \\ (m-1) / 2 & \text { if } m \text { odd }\end{array}\right.$.

Then

$$
\prod_{j=1}^{m}\left(1-x_{j}^{\beta_{j}}\right)^{1 / \beta_{j}}+\prod_{j=1}^{m} x_{j} \geq 1-\sum_{j=1}^{\xi(m)} \frac{\left(x_{2 j}^{\beta_{2 j}}-x_{2 j-1}^{\beta_{2 j-1}}\right)^{2}}{\beta_{2 j}} .
$$

Using the same methods as in Lemma 6, we get the following Lemma.

Lemma 7. Let $0>\beta_{1} \geq \beta_{2} \geq \cdots \geq \beta_{m}, m \geq 2$, let $x_{j}>1(j=$ $1,2, \ldots, m)$, and let $\xi(m)=\left\{\begin{array}{ll}m / 2 & \text { if } m \text { even } \\ (m-1) / 2 & \text { if } m \text { odd }\end{array}\right.$.

Then

$$
\prod_{j=1}^{m}\left(1-x_{j}^{\beta_{j}}\right)^{1 / \beta_{j}}+\prod_{j=1}^{m} x_{j} \geq 1-\sum_{j=1}^{\xi(m)} \frac{\left(x_{2 j}^{\beta_{2 j}}-x_{2 j-1}^{\beta_{2 j-1}}\right)^{2}}{\beta_{2 j}} .
$$

Now, we present some new refinements of inequalities (4) and (5).

Theorem 8. Let $a_{r j}>0, r=1,2, \ldots, n, j=1,2, \ldots, m, m \geq$ $2, n \geq 2$, let $0<\beta_{1} \leq \beta_{2} \leq \cdots \leq \beta_{m}, \sum_{j=1}^{m}\left(1 / \beta_{j}\right) \geq 1$, $a_{1 j}^{\beta_{j}}-\sum_{r=2}^{n} a_{r j}^{\beta_{j}}>0$, and let $\xi(m)=\left\{\begin{array}{ll}m / 2 & \text { if } m \text { even } \\ (m-1) / 2 & \text { if } m \text { odd }\end{array}\right.$.
Then

$$
\begin{aligned}
\prod_{j=1}^{m}\left(a_{1 j}^{\beta_{j}}-\sum_{r=2}^{n} a_{r j}^{\beta_{j}}\right)^{1 / \beta_{j}} \\
\leq \prod_{j=1}^{m} a_{1 j}-\sum_{r=2}^{n} \prod_{j=1}^{m} a_{r j} \\
\quad-\frac{a_{11} a_{12} \ldots a_{1 m}}{\xi(m)} \\
\quad \times \sum_{j=1}^{\xi(m)}\left\{\frac{1}{\max \left\{\beta_{2 j}, 1\right\}}\right.
\end{aligned}
$$

$$
\left.\times\left[\sum_{r=2}^{n}\left(\frac{a_{r(2 j)}^{\beta_{2 j}}}{\frac{a_{r(2 j-1)}^{\beta_{2 j-1}}}{a_{1(2 j)}^{\beta_{2 j}}}-\frac{a_{1(2 j-1)}^{\beta_{2 j-1}}}{\beta^{2}}}\right)\right]^{2}\right\}
$$

Proof. From the assumptions we find that

$$
0<\frac{\left(a_{1 j}^{\beta_{j}}-\sum_{r=2}^{n} a_{r j}^{\beta_{j}}\right)^{1 / \beta_{j}}}{\left(a_{1 j}^{\beta_{j}}\right)^{1 / \beta_{j}}}<1 \quad(j=1,2, \ldots, m) .
$$

Thus, by using Lemma 5 with a substitution $x_{j} \rightarrow\left(\left(a_{1 j}^{\beta_{j}}-\right.\right.$ $\left.\left.\sum_{r=2}^{n} a_{r j}^{\beta_{j}}\right) / a_{1 j}^{\beta_{j}}\right)^{1 / \beta_{j}}(j=1,2, \ldots, m)$ in (13), we obtain

$$
\begin{aligned}
& \prod_{j=1}^{m}\left(\frac{\sum_{r=2}^{n} a_{r j}^{\beta_{j}}}{a_{1 j}^{\beta_{j}}}\right)^{1 / \beta_{j}}+\prod_{j=1}^{m}\left(\frac{a_{1 j}^{\beta_{j}}-\sum_{r=2}^{n} a_{r j}^{\beta_{j}}}{a_{1 j}^{\beta_{j}}}\right)^{1 / \beta_{j}} \\
& \leq 1-\frac{1}{\xi(m)} \sum_{j=1}^{\xi(m)}\left\{\frac{1}{\max \left\{\beta_{2 j}, 1\right\}}\right. \\
& \times\left[\left(1-\frac{\sum_{r=2}^{n} a_{r(2 j)}^{\beta_{2 j}}}{a_{1(2 j)}^{\beta_{2 j}}}\right)\right. \\
& \left.\left.-\left(1-\frac{\sum_{r=2}^{n} a_{r(2 j-1)}^{\beta_{2 j-1}}}{a_{1(2 j-1)}^{\beta_{2 j-1}}}\right)\right]^{2}\right\} \\
& =1-\frac{1}{\xi(m)} \sum_{j=1}^{\xi(m)}\left\{\frac{1}{\max \left\{\beta_{2 j}, 1\right\}}\right. \\
& \left.\times\left[\sum_{r=2}^{n}\left(\frac{a_{r(2 j)}^{\beta_{2 j}}}{a_{1(2 j)}^{\beta_{2 j}}}-\frac{a_{r(2 j-1)}^{\beta_{2 j-1}}}{a_{1(2 j-1)}^{\beta_{2 j-1}}}\right)\right]^{2}\right\},
\end{aligned}
$$


which implies

$$
\begin{aligned}
& \prod_{j=1}^{m}\left(a_{1 j}^{\beta_{j}}-\sum_{r=2}^{n} a_{r j}^{\beta_{j}}\right)^{1 / \beta_{j}} \\
& \leq \prod_{j=1}^{m} a_{1 j}-\prod_{j=1}^{m}\left(\sum_{r=2}^{n} a_{r j}^{\beta_{j}}\right)^{1 / \beta_{j}} \\
& \quad-\frac{a_{11} a_{12} \ldots a_{1 m}}{\xi(m)} \\
& \quad \times \sum_{j=1}^{\xi(m)}\left\{\frac{1}{\max \left\{\beta_{2 j}, 1\right\}}\right. \\
& \left.\left.\times\left[\sum_{r=2}^{n}\left(\begin{array}{ll}
a_{r(2 j)}^{\beta_{2 j}} & \frac{a_{r(2 j-1)}^{\beta_{2 j-1}}}{a_{1(2 j)}^{\beta_{2 j}}}
\end{array}\right]^{a_{1(2 j-1)}^{\beta_{2 j-1}}}\right)\right]^{2}\right\} .
\end{aligned}
$$

On the other hand, we get from Lemma 3 that

$$
\prod_{j=1}^{m}\left(\sum_{r=2}^{n} a_{r j}^{\beta_{j}}\right)^{1 / \beta_{j}} \geq \sum_{r=2}^{n} \prod_{j=1}^{m} a_{r j} .
$$

Combining (26) and (27) yields immediately the desired inequality (23).

Theorem 9. Let $a_{r j}>0,0>\beta_{1} \geq \beta_{2} \geq \cdots \geq \beta_{m}, a_{1 j}^{\beta_{j}}-$ $\sum_{r=2}^{n} a_{r j}^{\beta_{j}}>0, r=1,2, \ldots, n, j=1,2, \ldots, m$, let $m \geq 2, n \geq 2$, and let $\xi(m)=\left\{\begin{array}{ll}m / 2 & \text { if } m \text { even } \\ (m-1) / 2 & \text { if } m \text { odd }\end{array}\right.$.

Then

$$
\begin{aligned}
\prod_{j=1}^{m}\left(a_{1 j}^{\beta_{j}}-\sum_{r=2}^{n} a_{r j}^{\beta_{j}}\right)^{1 / \beta_{j}} \geq \prod_{j=1}^{m} a_{1 j}-\sum_{r=2}^{n} \prod_{j=1}^{m} a_{r j} \\
\quad-a_{11} a_{12}, \ldots, a_{1 m} \\
\quad \times \sum_{j=1}^{\xi(m)}\left\{\frac{1}{\beta_{2 j}}\left[\sum_{r=2}^{n}\left(\frac{a_{r(2 j)}^{\beta_{2 j}}}{a_{1(2 j)}^{\beta_{2 j}}}-\frac{a_{r(2 j-1)}^{\beta_{2 j-1}}}{a_{1(2 j-1)}^{\beta_{2 j-1}}}\right)\right]^{2}\right\} .
\end{aligned}
$$

Inequality (28) is also valid for $\beta_{1}>0,0>\beta_{2} \geq \beta_{3} \geq \cdots \geq \beta_{m}$, $\sum_{j=1}^{m}\left(1 / \beta_{j}\right) \leq 1$.

Proof. The proof of Theorem 9 is similar to the one of Theorem 8, and we omit it.

\section{Applications}

In this section, we show two applications of the inequalities newly obtained in Section 2.

Firstly, we present a new refinement of inequality (6) by using Theorem 9.
Theorem 10. Let $t_{j}>0(j=1,2, \ldots, m), \beta_{1}>0,0>$ $\beta_{2} \geq \beta_{3} \geq \cdots \geq \beta_{m}, \sum_{j=1}^{m}\left(1 / \beta_{j}\right)=1$, let $f_{j}(x)(j=$ $1,2, \ldots, m)$ be positive integrable functions defined on $[a, b]$ with $t_{j}^{\beta_{j}}-\int_{a}^{b} f_{j}^{\beta_{j}}(x) d x>0$, and let $\xi(m)=\left\{\begin{array}{cc}m / 2 & \text { if } m \text { even } \\ (m-1) / 2 & \text { if } m \text { odd }\end{array}\right.$. Then

$$
\begin{aligned}
\prod_{j=1}^{m}\left(t_{j}^{\beta_{j}}-\int_{a}^{b} f_{j}^{\beta_{j}}(x) d x\right)^{1 / \beta_{j}} \\
\quad \geq \prod_{j=1}^{m} t_{j}-\int_{a}^{b} \prod_{j=1}^{m} f_{j}(x) d x \\
\quad-t_{1} t_{2}, \ldots, t_{m} \\
\quad \times \sum_{j=1}^{\xi(m)}\left[\frac{1}{\beta_{2 j}} \int_{a}^{b}\left(\frac{f_{2 j}^{\beta_{2 j}}(x)}{t_{2 j}^{\beta_{2 j}}}-\frac{f_{2 j-1}^{\beta_{2 j-1}}(x)}{t_{2 j-1}^{\beta_{2 j-1}}}\right) d x\right]^{2} .
\end{aligned}
$$

Proof. For any positive integer $n$, we choose an equidistant partition of $[a, b]$ as

$$
\begin{gathered}
a<a+\frac{b-a}{n}<\cdots<a+\frac{b-a}{n} k \\
<\cdots<a+\frac{b-a}{n}(n-1)<b, \\
x_{i}=a+\frac{b-a}{n} i, \quad i=0,1, \ldots, n, \\
\Delta x_{k}=\frac{b-a}{n}, \quad k=1,2, \ldots, n .
\end{gathered}
$$

Since $t_{j}^{\beta_{j}}-\int_{a}^{b} f_{j}^{\beta_{j}}(x) d x>0(j=1,2, \ldots, m)$, it follows that

$$
\begin{array}{r}
t_{j}^{\beta_{j}}-\lim _{n \rightarrow \infty} \sum_{k=1}^{n} f_{j}^{\beta_{j}}\left(a+\frac{k(b-a)}{n}\right) \frac{b-a}{n}>0 \\
(j=1,2, \ldots, m) .
\end{array}
$$

Therefore, there exists a positive integer $N$ such that

$$
t_{j}^{\beta_{j}}-\sum_{k=1}^{n} f_{j}^{\beta_{j}}\left(a+\frac{k(b-a)}{n}\right) \frac{b-a}{n}>0,
$$

for all $n>N$ and $j=1,2, \ldots, m$. 
Moreover, for any $n>N$, it follows from Theorem 9 that

$$
\begin{aligned}
\prod_{j=1}^{m}\left[t_{j}^{\beta_{j}}-\sum_{k=1}^{n} f_{j}^{\beta_{j}}\left(a+\frac{k(b-a)}{n}\right) \frac{b-a}{n}\right]^{1 / \beta_{j}} \\
\geq \prod_{j=1}^{m} t_{j}^{\beta_{j}}-\sum_{k=1}^{n}\left(\prod_{j=1}^{m} f_{j}\left(a+\frac{k(b-a)}{n}\right)\right) \\
\quad \times\left(\frac{b-a}{n}\right)^{1 / \beta_{1}+1 / \beta_{2}+\cdots+1 / \beta_{m}}-t_{1} t_{2}, \ldots, t_{m} \sum_{j=1}^{\xi(m)} \frac{1}{\beta_{2 j}} \\
\times\left[\sum _ { k = 1 } ^ { n } \left(\frac{1}{t_{2 j}^{\beta_{2 j}}} f_{2 j}^{\beta_{2 j}}\left(a+\frac{k(b-a)}{n}\right) \frac{b-a}{n}\right.\right. \\
\left.\left.\quad-\frac{1}{t_{2 j-1}^{\beta_{2 j-1}}} f_{2 j-1}^{\beta_{2 j-1}}\left(a+\frac{k(b-a)}{n}\right) \frac{b-a}{n}\right)\right]^{2} .
\end{aligned}
$$

Noting that

$$
\sum_{j=1}^{m} \frac{1}{\beta_{j}}=1,
$$

we get

$$
\begin{aligned}
& \prod_{j=1}^{m}\left[t_{j}^{\beta_{j}}-\sum_{k=1}^{n} f_{j}^{\beta_{j}}\left(a+\frac{k(b-a)}{n}\right) \frac{b-a}{n}\right]^{1 / \beta_{j}} \\
& \geq \prod_{j=1}^{m} t_{j}^{\beta_{j}}-\sum_{k=1}^{n}\left(\prod_{j=1}^{m} f_{j}\left(a+\frac{k(b-a)}{n}\right)\right)\left(\frac{b-a}{n}\right) \\
& -t_{1} t_{2}, \ldots, t_{m} \sum_{j=1}^{\xi(m)} \frac{1}{\beta_{2 j}} \\
& \times\left[\sum _ { k = 1 } ^ { n } \left(\frac{1}{t_{2 j}^{\beta_{2 j}}} f_{2 j}^{\beta_{2 j}}\left(a+\frac{k(b-a)}{n}\right) \frac{b-a}{n}\right.\right. \\
& \left.\left.\quad-\frac{1}{t_{2 j-1}^{\beta_{2 j-1}}} f_{2 j-1}^{\beta_{2 j-1}}\left(a+\frac{k(b-a)}{n}\right) \frac{b-a}{n}\right)\right]^{2} .
\end{aligned}
$$

In view of the assumption that $f_{j}(x)(j=1,2, \ldots, m)$ are positive Riemann integrable functions on $[a, b]$, we find that $\prod_{j=1}^{m} f_{j}(x)$ and $f_{j}^{\lambda_{j}}(x)$ are also integrable on $[a, b]$. Letting $n \rightarrow \infty$ on both sides of inequality (36), we get the desired inequality (29).

Next, we give a new refinement of inequality (7) by using Theorem 8 .

Theorem 11. Let $t_{j}>0(j=1,2, \ldots, m), 0<\beta_{1} \leq \beta_{2} \leq$ $\cdots \leq \beta_{m}, \sum_{j=1}^{m}\left(1 / \beta_{j}\right)=1, m \geq 2$, and let $f_{j}(x)(j=$
$1,2, \ldots, m)$ be positive integrable functions defined on $[a, b]$ with $t_{j}^{\beta_{j}}-\int_{a}^{b} f_{j}^{\beta_{j}}(x) d x>0$, and let $\xi(m)=\left\{\begin{array}{cc}m / 2 & \text { if } m \text { even } \\ (m-1) / 2 & \text { if } m \text { odd }\end{array}\right.$.

Then

$$
\begin{gathered}
\prod_{j=1}^{m}\left(t_{j}^{\beta_{j}}-\int_{a}^{b} f_{j}^{\beta_{j}}(x) d x\right)^{1 / \beta_{j}} \leq \prod_{j=1}^{m} t_{j}-\int_{a}^{b} \prod_{j=1}^{m} f_{j}(x) d x \\
-\frac{t_{1} t_{2}, \ldots, t_{m}}{\xi(m)} \\
\times \sum_{j=1}^{\xi(m)}\left\{\frac{1}{\beta_{2 j}}\right. \\
\left.\times\left[\int_{a}^{b}\left(\frac{f_{2 j}^{\beta_{2 j}}(x)}{t_{2 j}^{\beta_{2 j}}}-\frac{f_{2 j-1}^{\beta_{2 j-1}}(x)}{t_{2 j-1}^{\beta_{2 j-1}}}\right) d x\right]^{2}\right\} .
\end{gathered}
$$

Proof. The proof of Theorem 11 is similar to the one of Theorem 10, and we omit it.

\section{Acknowledgments}

The authors would like to thank the reviewers and the editors for their valuable suggestions and comments. This work was supported by the Fundamental Research Funds for the Central Universities (Grant no. 13ZD19).

\section{Conflict of Interests}

The authors declare that there is no conflict of interests regarding the publication of this paper.

\section{References}

[1] Y. Aczél, "Some general methods in the theory of functional equations in one variable. New applications of functional equations," Uspekhi Matematicheskikh Nauk, vol. 11, no. 3, pp. 3-68, 1956 (Russian).

[2] E. F. Beckenbach and R. Bellman, Inequalities, Springer, Berlin, Germany, 1983.

[3] G. Farid, J. Pečarić, and A. Ur Rehman, "On refinements of Aczél, Popoviciu, Bellman's inequalities and related results," Journal of Inequalities and Applications, vol. 2010, Article ID 579567, 2010.

[4] Z. Hu and A. Xu, "Renements of Aczél and Bellman's inequalities," Computers \& Mathematics with Applications, vol. 59, no. 9, pp. 3078-3083, 2010.

[5] F. Mirzapour, A. Morassaei, and M. S. Moslehian, "More on operator Bellman inequality," Quaestiones Mathematicae, vol. 37, no. 1, pp. 9-17, 2014.

[6] D. S. Mitrinović, J. E. Pečarić, and A. M. Fink, Classical and New Inequalities in Analysis, Kluwer Academic Publishers, Dordrecht, The Netherlands, 1993.

[7] B. Mond, J. Pečarić, J. Šunde, and S. Varošanec, "Operator versions of some classical inequalities," Linear Algebra and Its Applications, vol. 264, no. 1, pp. 117-126, 1997. 
[8] A. Morassaei, F. Mirzapour, and M. S. Moslehian, "Bellman inequality for Hilbert space operators," Linear Algebra and its Applications, vol. 438, no. 10, pp. 3776-3780, 2013.

[9] M. S. Moslehian, “Operator Aczél inequality, Linear Algebra and Its Applications, vol. 434, no. 8, pp. 1981-1987, 2011.

[10] T. Popoviciu, "On an inequality," Gazeta Matematica si Fizica. Seria A, vol. 11, no. 64, pp. 451-461, 1959 (Romanian).

[11] J. Tian, "A sharpened and generalized version of Aczel-VasicPecaric inequality and its application," Journal of Inequalities and Applications, vol. 2013, article 497, 2013.

[12] J. Tian and X.-M. Hu, "A new reversed version of a generalized sharp Hölder's inequality and its applications," Abstract and Applied Analysis, vol. 2013, Article ID 901824, 9 pages, 2013.

[13] J. Tian, "Reversed version of a generalized Aczél's inequality and its application," Journal of Inequalities and Applications, vol. 2012, article 202, 2012.

[14] J.-F. Tian and S. Wang, "Refinements of generalized aczél's inequality and bellman's inequality and their applications," Journal of Applied Mathematics, vol. 2013, Article ID 645263, 6 pages, 2013.

[15] P. M. Vasić and J. E. Pečarić, "On the Jensen inequality for monotone functions," Analele Universitatii din Timisoara, Seria Stiinte Matematice, vol. 17, no. 1, pp. 95-104, 1979.

[16] P. M. Vasić and J. E. Pečarić, "On the Hölder and some related inequalities," Mathematica, vol. 24, no. 47, pp. 95-103, 1982.

[17] S. Vong, "On a generalization of Aczél's inequality," Applied Mathematics Letters, vol. 24, no. 8, pp. 1301-1307, 2011.

[18] S. Wu, "Some improvements of Aczél's inequality and Popoviciu's inequality," Computers \& Mathematics with Applications, vol. 56, no. 5, pp. 1196-1205, 2008.

[19] S. Wu and L. Debnath, "Generalizations of Aczel's inequality and Popoviciu's inequality," Indian Journal of Pure and Applied Mathematics, vol. 36, no. 2, pp. 49-62, 2005.

[20] S. Wu and L. Debnath, "A new generalization of Aczél's inequality and its applications to an improvement of Bellman's inequality," Applied Mathematics Letters, vol. 21, no. 6, pp. 588593, 2008.

[21] X. Zhou, "Some generalizations of Aczél, Bellman's inequalities and related power sums," Journal of Inequalities and Applications, vol. 2012, article 130, 2012. 


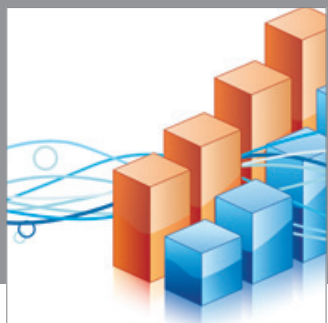

Advances in

Operations Research

mansans

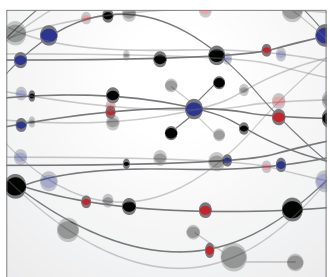

The Scientific World Journal
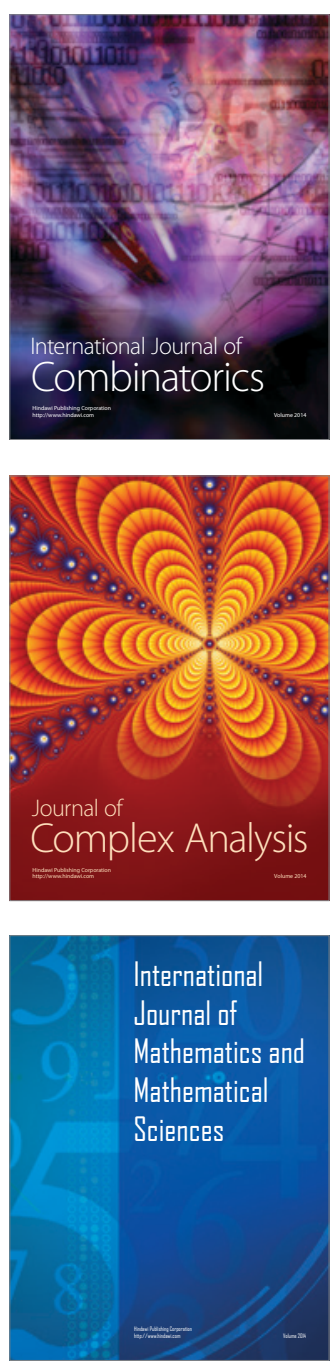
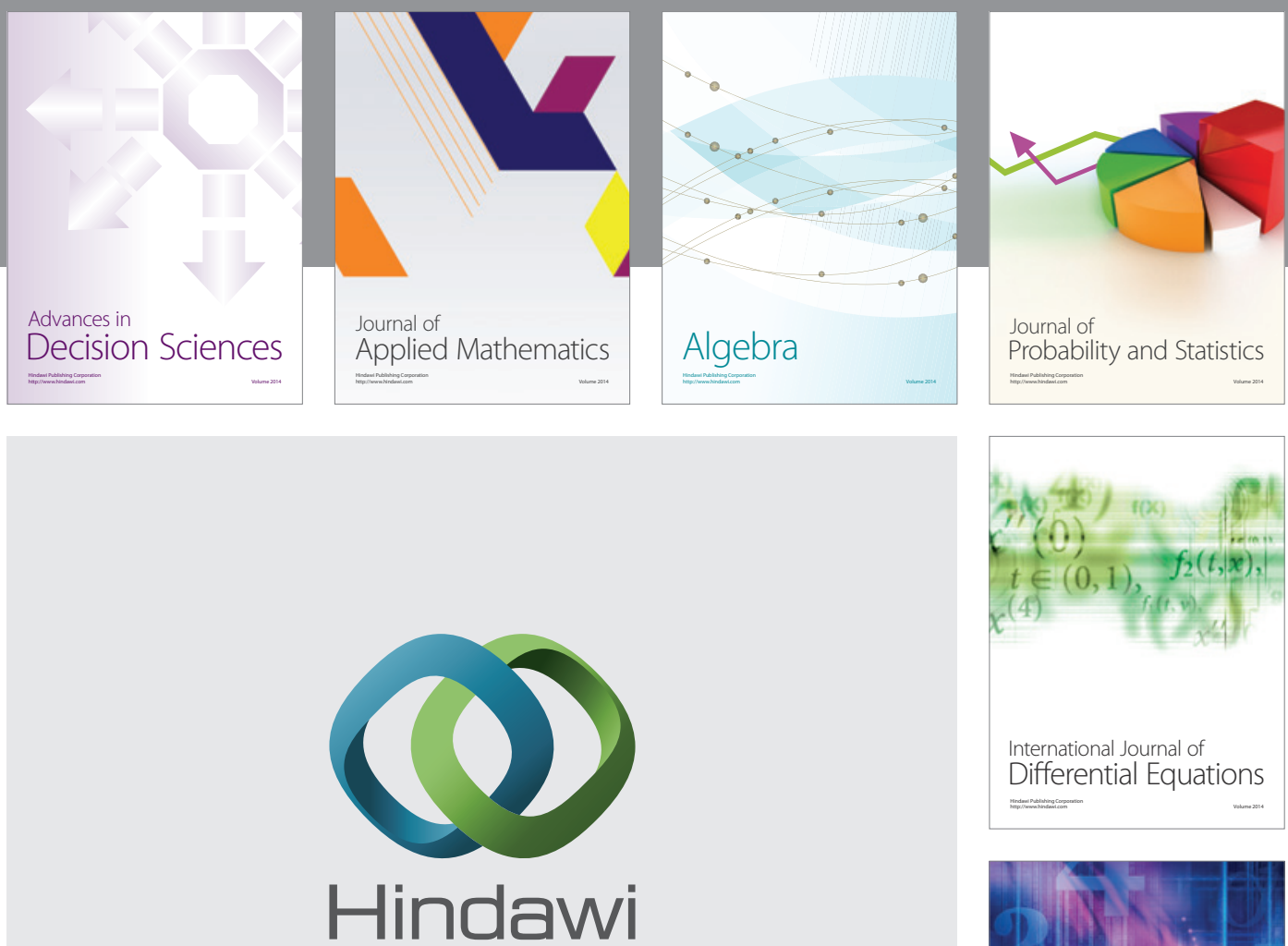

Submit your manuscripts at http://www.hindawi.com
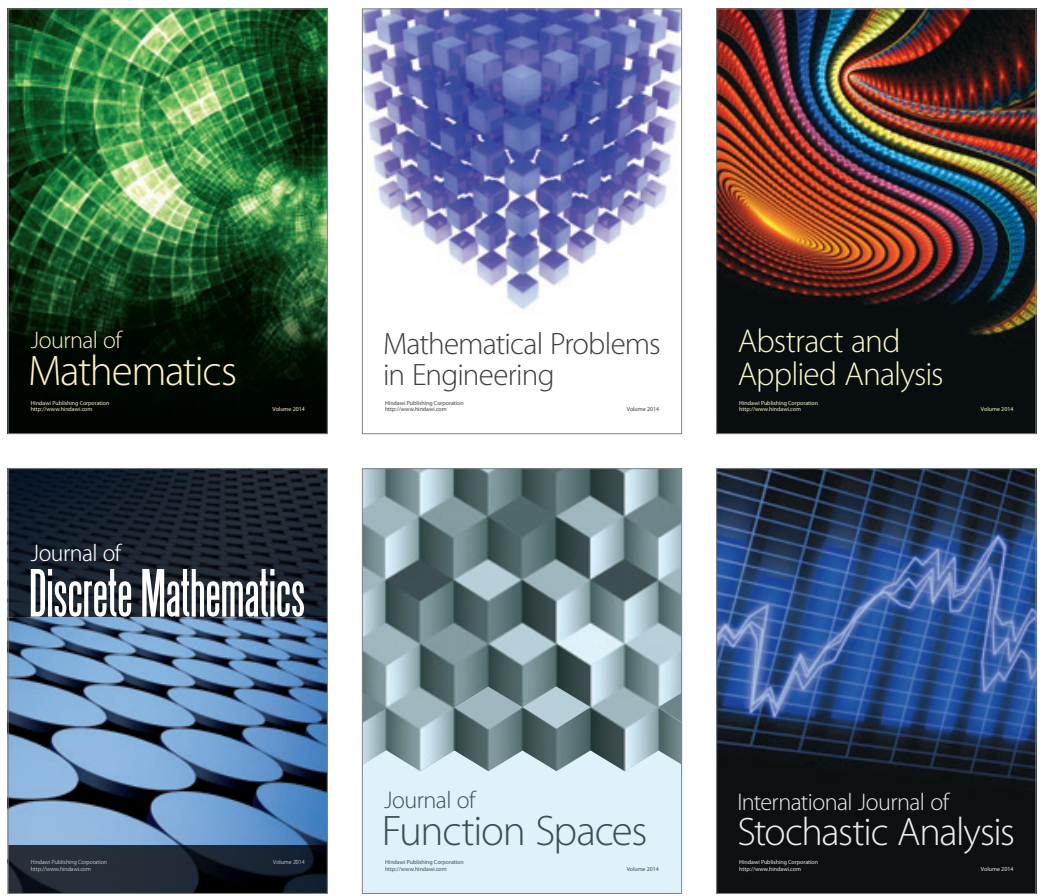

Journal of

Function Spaces

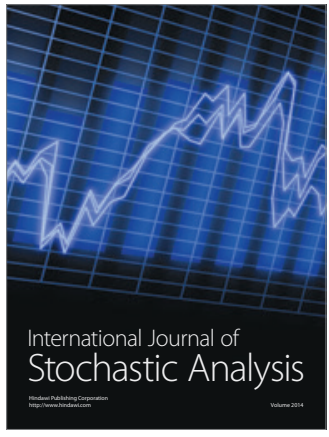

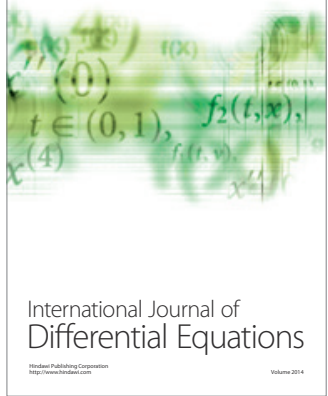
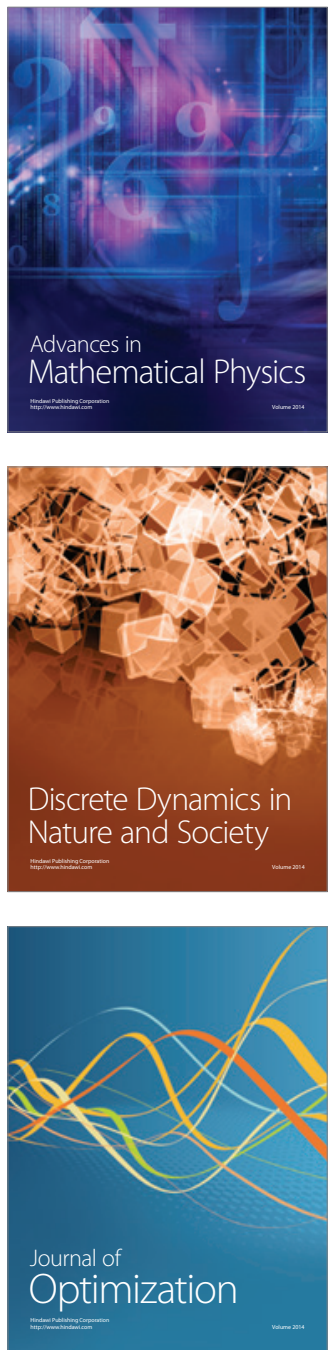